\title{
In Situ Assessment of the Liver Microcirculation in Mechanically Ventilated Rats using Sidestream Dark-Field Imaging
}

\author{
V. ČERNÝ, Z. TUREK, R. PAŘÍZKOVÁ
}

Department of Anesthesiology and Intensive Care Medicine, Charles University in Prague, Faculty of Medicine in Hradec Králové, University Hospital, Hradec Králové, Czech Republic

Received July 15, 2007

Accepted October 26, 2007

On-line January 17, 2008

\section{Summary}

Assessment of hepatic microcirculation by on-line visualization has been impossible for a long time. Sidestream dark-field (SDF) imaging is a relatively new method allowing direct visualization of both mucosal microcirculation and surface layers microcirculation of solid organs using hand-held probe for direct contact with target tissue. The aim of this study was to evaluate the feasibility of studying the rat hepatic microcirculation in situ by SDF imaging. The liver lobes were left in situ, and images were obtained using SDF imaging on the surface of the liver via upper midline laparotomy. Images were captured intermittently during 10-sec apnoea and recorded. The microvascular parameters were compared with previous validation studies. Clear high contrast SDF images were successfully obtained. Quantitative analysis revealed a mean FSD (functional sinusoidal density) of $402 \pm 15$ $\mathrm{cm} / \mathrm{cm}^{2}$, a sinusoidal diameter of $10.2 \pm 0.5 \mu \mathrm{m}$ and postsinusoidal venular diameter of $33.9 \pm 13 \mu \mathrm{m}$. SDF imaging is a suitable noninvasive method for accurate quantification of the basic microcirculatory parameters of the liver in situ without a need to exteriorize the liver lobes. This method seems to be applicable in animal studies with possibility to use SDF imaging also intraoperatively, providing unique opportunity to study liver microcirculation during various experimental and clinical settings.

\section{Key words}

SDF imaging - Liver perfusion - Intermittent positive pressure ventilation • Rats

\section{Corresponding author}

V. Černý, Department of Anesthesiology and Intensive Care Medicine, University Hospital Hradec Kralové, Sokolská 581, 50005 Hradec Kralové, Czech Republic. E-mail cernyvla@fnhk.cz

\section{Introduction}

A wide variety of insults affecting the liver (e.g. sepsis, ischemia-reperfusion injury or hypovolemia) can induce the changes in the microvascular regulation, resulting in regions with compromised perfusion leading to the various clinical consequences (Clemens et al. 2005). Although the regulatory mechanisms of hepatic sinusoidal blood flow has not been fully understood, previous animal studies have demonstrated that effective restoration of microvascular perfusion is an essential determinant of the liver function recovery (Chun et al. 1994). For many years, intravital microscopy (IVM) of the liver was the only powerful experimental method to investigate hepatic microcirculation under physiological and pathological conditions allowing direct visualization of the microcirculatory network of the liver. Orthogonal polarization spectral (OPS) imaging and its successor sidestream dark-field (SDF) imaging are relatively new, progressively developing, noninvasive optical methods with a potential to directly visualize microcirculation based on very similar principles, which have been described in details previously (Groner et al. 1999, Schiessler et al. 2002, De Backer 2003, Ince 2005). Briefly, in OPS imaging green $(550 \pm 70 \mathrm{~nm})$ polarized light, guided through a system of lenses, illuminates the target tissue to achieve optimal imaging of microcirculation because at this wavelength oxy- and deoxy-hemoglobin absorb the light equally. The light reflected at the surface is eliminated by orthogonal polarizer (analyzer) and does not form the image. The light after undergoing multiple scattering becomes depolarized and passes through the polarizer to create an 
image of the underlying microcirculation. SDF imaging is based on slightly different principles as compared with OPS technology. Light-emitting diodes (LEDs) arranged in a ring formation at the tip of the light guide emit green light $(540 \pm 50 \mathrm{~nm})$ which directly illuminates the tissue microcirculation. The illuminating light source is optically isolated from the emission light path in the core of the light guide (Fig. 1). Hence, SDF technology provides improved resolution and clarity of the images compared to OPS imaging. Both SDF and OPS technology have been implemented in a hand-held videomicroscope convenient for both experimental and clinical conditions. Validation studies which have been reviewed recently have shown a statistically significant agreement of the data obtained from new optical technologies and IVM (Černý et al. 2006). The SDF imaging method could represent a unique tool for assessment of hepatic microcirculation both in experimental and human studies.

The purpose of the experimental study was designed to elucidate whether SDF imaging in anesthetized mechanically ventilated rats can be used for direct imaging of hepatic sinusoidal perfusion and quantitative assessment of the basic physiological hepatic microcirculatory parameters in vivo. The results were compared with current available validation studies and possible further clinical applications are discussed as well.

\section{Methods}

\section{Animals}

All experimental procedures were performed after Ethical Board approval in accordance with Czech legislation on the protection of animals. Nine male Wistar rats (Bio-Test, Konarovice, Czech Republic), weighing 310-350 g, were included in the study. They were housed in groups of two in a standard cage at $21{ }^{\circ} \mathrm{C}$ in a $12 \mathrm{~h}$ dark/12 h light cycle and were supplied with laboratory chow (Velaz, Prague, Czech Republic) and tap water ad libitum. After one week acclimatization period, the rats were enrolled into the study.

\section{Anesthesia and surgical preparation}

After overnight fasting with unrestricted access to tap water, the rats were anesthetized with an intraperitoneal induction dose of pentobarbital (Nembutal, Abbott Laboratories, Chicago, IL, USA; $50 \mathrm{mg} / \mathrm{kg} \mathrm{bw}$ ). The animals were placed in a supine position on an operating table, rectal temperature was kept at $36.5-37.5{ }^{\circ} \mathrm{C}$ by a heating lamp. The carotid artery and femoral vein were cannulated with polyethylene catheter $24 \mathrm{G}$ for continuous blood pressure monitoring, continuous infusion of normal saline $(20 \mathrm{ml} / \mathrm{kg} / \mathrm{h})$ and administration of additional anesthesia and muscle relaxant pipecuronium bromide (Arduan, Richter Ltd, Budapest, Hungary; $0.2 \mathrm{mg} / \mathrm{kg}$ of body weight) as necessary. The animals were tracheotomized (Vasocan 14G, B. Braun Melsungen AG, Germany) and mechanically ventilated (ventilator Harvard Scientific, Boston, USA, ventilatory setting: tidal volume $10 \mathrm{ml} / \mathrm{kg}$, PEEP $2 \mathrm{~cm} \mathrm{H}_{2} \mathrm{O}$, respiratory rate 60-70/min, $\mathrm{FiO}_{2}$ 0.4). Mean arterial blood pressure (MAP) and rectal temperature were recorded every $5 \mathrm{~min}$ in each animal throughout the study.

Different surgical model from those widely used in previous animal studies (Kondo et al. 1998, Langer et al. 2001, Marzi et al. 1990, Menger et al. 1991, Terajima et al. 1999, Vollmar et al. 1994) was employed for assessment of hepatic microcirculatory changes using SDF technology in rat. After having shaved their abdomen an upper midline laparotomy was performed to visualize left and right liver lobes. Liver lobes were kept in situ without any mechanical manipulation throughout the study. Then the wound was covered by sterile $37^{\circ} \mathrm{C}$ warmed normal saline-saturated cotton swabs to minimize dehydration and loss of body heat. A waiting period of $20 \mathrm{~min}$ followed to confirm the stability of arterial blood pressure and rectal temperature (Langer et al. 2000). SDF imaging was then performed according to protocol.

\section{Sidestream dark-field imaging procedure}

To minimize artificial pressure and movement artifacts, which is crucial (Lindert et al. 2002), the SDF imaging probe (MicroScan Video Microscope, Microvision Medical, Inc., Amsterdam, The Netherlands) was attached to a custom made (Arrow International Czech Republic, a.s.) flexible arm with special adapter allowing micromovement of the SDF probe in various axes according to inclination of the flexible arm (Fig. 2) and proper stabilization of the probe at the same time. In an effort to objectify the SDF imaging of microcirculation as much as possible, the following methodology was established: once a sector for imaging was selected, the SDF imaging probe was placed approximately $0.5 \mathrm{~mm}$ above the target tissue using a flexible arm. Then the arm was fixed and the probe was 


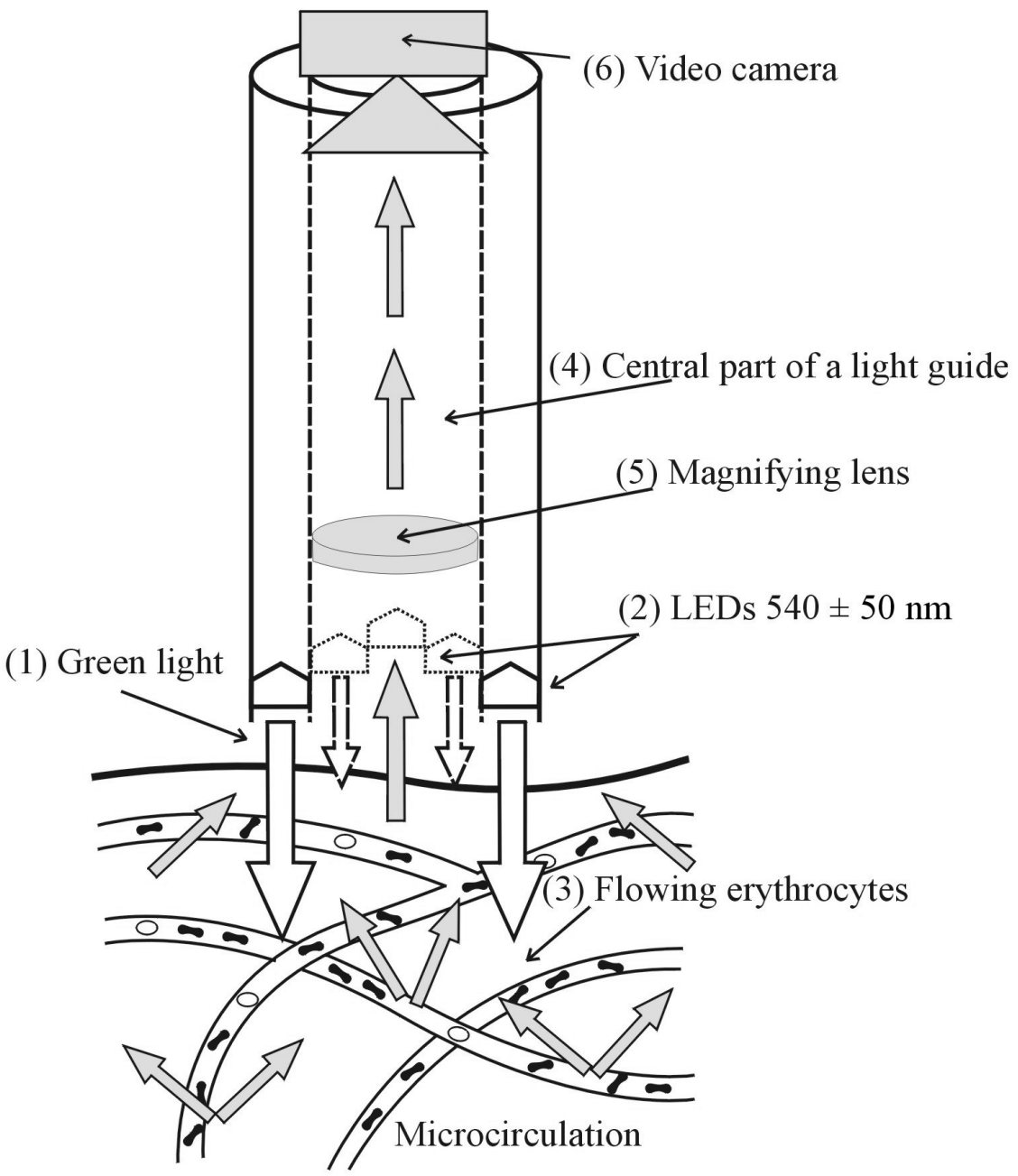

Fig. 1. Sidestream dark-field (SDF) imaging, an optical scheme. (1) Green light is emitted by (2) peripheral $540 \pm 50 \mathrm{~nm}$ lightemitting diodes (LEDs) toward tissue arranged in a circle at the end of the light guide. The microcirculation is directly illuminated from the side by green light absorbed by hemoglobin of erythrocytes which are observed as (3) dark moving cells. The imaging, central part, of the light guide (4) is optically isolated from the LEDs. A Magnifying lens (5) projects the image onto a camera (6). covered with plastic and the lens was moved towards tissue by the adapter for micromovement. Immediately after the first contact with the investigated organ - liver lobe, the focus ring of the probe was used to bring the proper layer into focus to create sharp and high contrast image appropriate for later off-line analysis. The sites of interests on the surface of the left and right liver lobe were selected randomly. In order to eliminate movement artifacts and lateral movement of the liver caused by mechanical ventilation at once and to keep the liver lobes in situ, all SDF images were recorded during $10 \mathrm{~s}$ lasting apnea (turning off ventilator). Each captured sequence was followed by $2 \mathrm{~min}$ of stabilizing period. Any exposed tissues, apart from those covered by SDF imaging probe at the given moment, were intermittently moisturized using warmed sterile normal saline at $37^{\circ} \mathrm{C}$. SDF images were obtained from three different areas within the site of interest as recommended recently (Boerma et al. 2005). All SDF imaging data of the microcirculation were digitally recorded. At the end of the experiment the animal was killed by an overdose of pentobarbital.

\section{Off-line analysis}

Off-line selection of the most stable clips with clear images for final analysis was performed. To summarize, a total of 18 selected clips ( 6 clips of each area) of $10 \mathrm{~s}$ were analyzed per each animal. The final on screen magnification of the images obtained with the SDF imaging device was 325 times original. Basic microcirculatory parameters such as functional sinusoidal density (FSD), sinusoidal diameter and postsinusoidal venular diameter were obtained using AVA V1.0 software (AMC, University of Amsterdam, The Netherlands).

The following parameters were analyzed offline: 1) functional sinusoidal density (FSD) given in $\mathrm{cm} / \mathrm{cm}^{2} ; 2$ ) the diameter of liver sinusoids of the midzonal segment of a liver acinus given in $\mu m ; 3)$ the diameter of postsinusoidal venules given in $\mu m ; 4)$ small $(<15 \mu \mathrm{m}$ in diameter) vessel rate $\mathrm{x} 100$ given in $\%$

\section{Statistics}

The descriptive data are presented as mean 


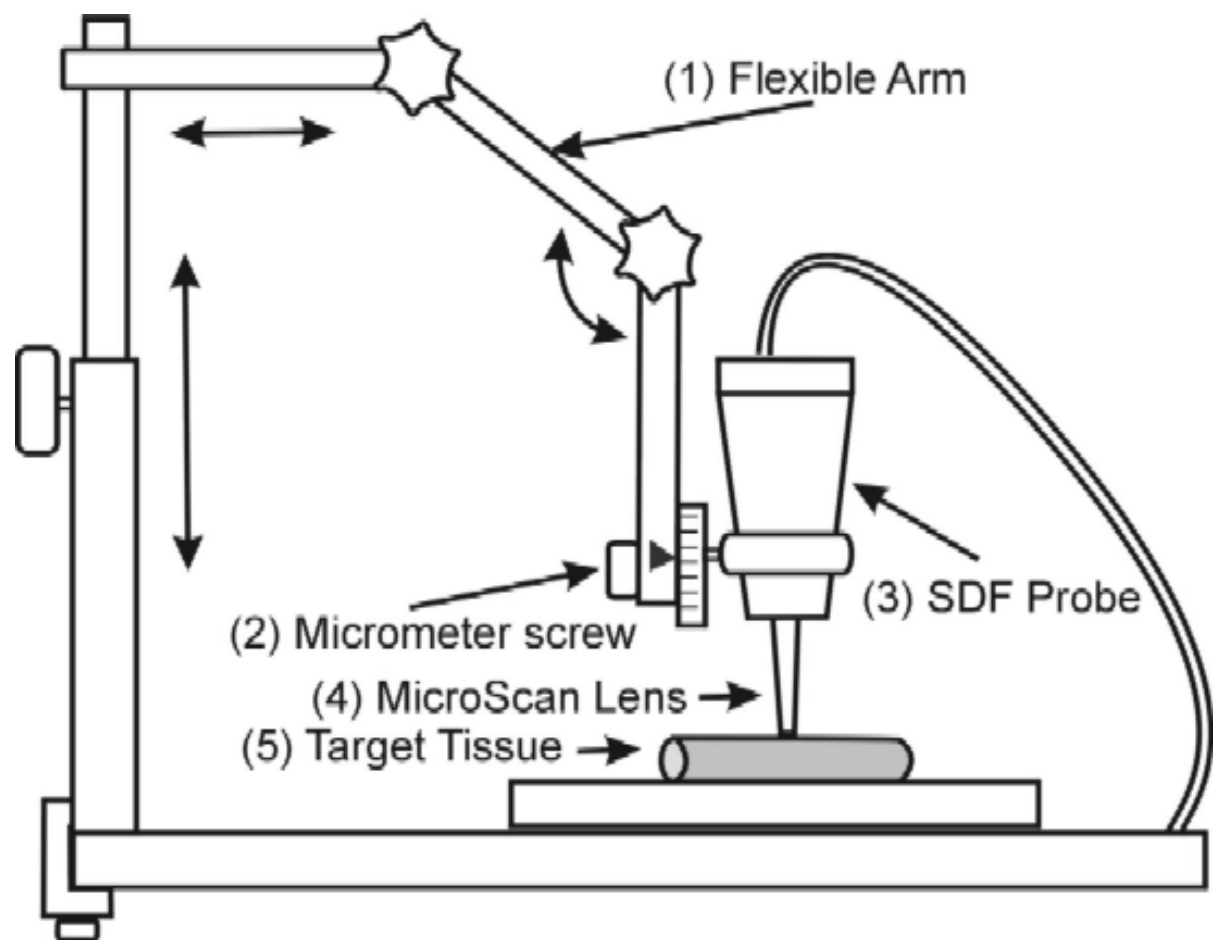

Fig. 2. A scheme of the stabilizing and fixation device for SDF imaging probe. (1) Flexible arm allowing horizontal, vertical and rotating movements ends with a special adapter with (2) micrometer screw for movement of the SDF probe (3) with MicroScan lens (4) within the range of $0.5 \mathrm{~mm}$ towards the target tissue (5).
(95\% confidence interval of mean; S.D. - standard deviation). Statistical analyses were performed with the use of SIGMASTAT 2.0 (Jandel Scientific, San Rafael, CA, USA).

\section{Results}

Main cardiovascular parameters were stable throughout the study with MAP of $100 \pm 6 \mathrm{~mm} \mathrm{Hg}$. No circulatory instability was observed during on-line SDF imaging in short-term apnea. With special custom made fixation device for the SDF probe, clear high contrast images were successfully obtained from the surface of the rat liver lobes in situ using SDF imaging technology. The morphological structures of hepatic microvasculature were clearly identified and it was possible to perform basic off-line quantitative measurements of microcirculatory parameters in mechanically ventilated rat. Typical images with characteristic structure of a liver acinus surface captured using SDF imaging are shown (Fig. 3). Results are given as mean (95\% confidence interval of mean; S.D.). The mean FSD obtained from the surface of the liver was $402(399-406 ; 14.4) \mathrm{cm} / \mathrm{cm}^{2}$, mean sinusoidal diameter was $10.2(10.1-10.3 ; 0.36) \mu \mathrm{m}$ and postsinusoidal venular diameter 34.0 (30.0-38.0; 13.2) $\mu \mathrm{m}$. The percentage analysis of small vessels (small vessel rate) has shown a value of 96.2 (94.0-98.4.7) \%.

\section{Discussion}

This study has confirmed the reliability and accuracy of the SDF imaging when assessing hepatic microcirculation. The values of basic microcirculatory parameters are compatible with those published in validation studies previously (Langer et al. 2000). SDF imaging optical technology is a relatively new noninvasive method for direct on-line visualization of microcirculation without a need to use phototoxic fluorescent dyes (Saetzler et al. 1997), which allows the application of this method both in experimental and clinical studies.

On-line visualization and recording of hepatic surface microcirculation was performed during short-term apneic pause (8-10 s) to avoid exteriorization of the liver lobe minimizing tissue injury and to simulate potentially real clinical conditions when using SDF imaging intraoperatively. The effect of this short apneic pause on hepatic microcirculation in rat cannot be excluded but no hemodynamic instability during monitoring MAP and heart rate was observed and the results were not different from those published previously (Langer et al. 2000, 2001).

Regulation of liver microcirculation with regard to dual circulation is very complex. The hepatic artery and the portal vein circulation join to perfuse the hepatic sinusoids. Although the sinusoids, like capillaries in other vascular beds, do not contain vascular smooth muscle 


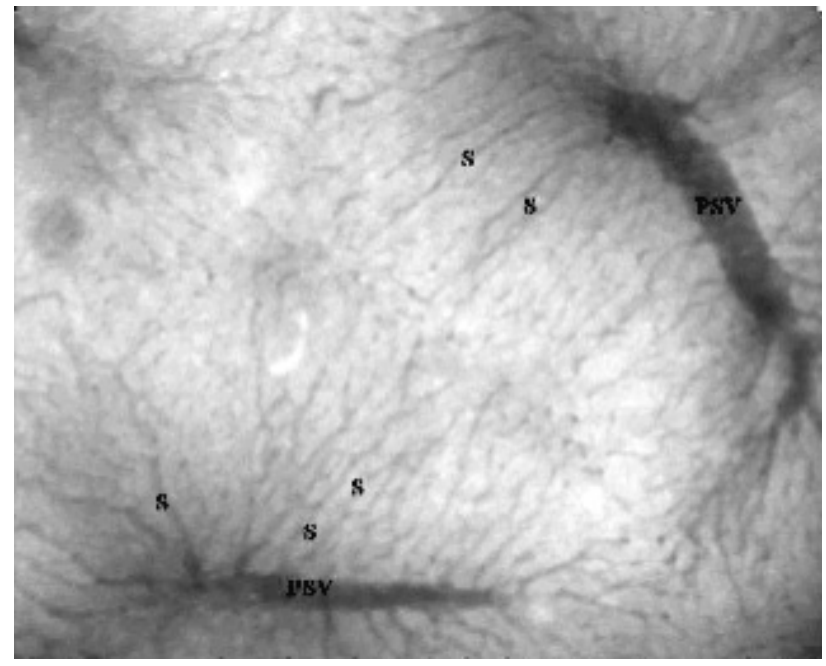

Fig. 3. SDF representative image of the rat liver microcirculation. Sidestream dark-field imaging of the the rat hepatic microcirculation. Objective $5 x$, on screen $325 x$. S - liver sinusoids, PSV - postsinusoidal venules

cells, it is commonly accepted that they are subject to active constriction (Zhang et al. 1994, 1995) mediated by specialized contractile pericytes called the hepatic stellate cells (HSC) (Bauer et al. 2000). Under physiological conditions, HSC contract in response to endogenous endothelins, but not $\alpha$-adrenergic agonists such as phenylephrine and relax in response to nitric oxide (NO) and carbon monooxide (Zhang et al. 1995). Endothelin expression is upregulated during endotoxemia, hypoxia, or ischemia (Sonin et al. 1999). However, the mechanisms by which endotoxemia or ischemia induce hepatic microcirculatory dysfunction are not fully understood. Thus accurate sinusoidal diameter measurement is of special importance both for future experimental and clinical studies. Our study demonstrates high accuracy in sinusoidal diameter visualization and measurement when using SDF imaging compared to previous validation studies mentioned above. The value of FSD reflects nutritional tissue perfusion and it is indirect parameter of oxygen delivery to the tissue (Harris et al. 1996). In our opinion, determination of FSD represents the preferable method for the SDF imaging technique, because determination of sinusoidal perfusion rate is impossible due to the basic principle of the method that does not allow the visualization of sinusoids not perfused with red blood cells.

Another modality affecting splanchnic microcirculation is artificial pulmonary ventilation with intermittent positive pressure ventilation (IPPV) which is accompanied by decreased cardiac output (CO) (Saner et al. 2006, Marini et al. 1980) and alteration of microcirculation in connection with decreased $\mathrm{CO}$ was also described (De Backer et al. 2004). Though IPPV is a component of the protocols in a number of experimental and clinical studies using the techniques of OPS or SDF imaging, the possible effect of IPPV is not mentioned in the interpretation of the results. The value of positive end-expiratory pressure (PEEP) is another measured variable that must be taken into account when assessing and interpreting changes at the microcirculatory level. As published previously, fluid resuscitation can reverse the undesirable effect of PEEP on $\mathrm{CO}$, but only partial correction of the negative effect of PEEP on mesenteric blood flow can be achieved (Love et al. 1995). Stable value of PEEP $2 \mathrm{~cm}$ of $\mathrm{H}_{2} \mathrm{O}$ was maintained throughout presented study so that PEEP is not supposed to be a fundamental variable affecting significantly hepatic microcirculation in this study.

Despite further technological improvements several limitations of this method remain (Lindert et al. 2002). Artificial pressure of the SDF probe and lateral movement of the tissue are the most important factors affecting accuracy of the measurement of microcirculatory parameters. Special custom-made device allowing micromovement of the probe, standardized procedure of the visualization with image focusing and short apneic pause were used in this study to minimized artifacts mentioned above, however, the possibility to avoid any micromovements of the probe at clinical settings is limited. Majority of microcirculatory parameters has to be analyzed off-line due to software limitation, further software upgrade will be desired in the course of putting the method into clinical practice.

The technology of SDF imaging has been incorporated into a small hand-held improved videomicroscope, which can be used in various clinical setting. Human studies using noninvasive optical technologies OPS or SDF imaging have been mainly conducted on nailfold (Mathura et al. 2001), brain (Pennings et al. 2006, Mathura and Ince 2000), cutis of term and preterm infants (Genzel-Boroviczeny et al. 2002), sublingual mucosa in critically ill patients (Sakr et al. 2004) and liver during liver resection and transplant surgery (Puhl et al. 2003, 2005). Due to high quality of the SDF images comparable with those obtained via IVM, their accurate quantitative analysis and easy manipulation, SDF technology should be considered as very useful and promising research tool for understanding of pathophysiology of liver microcirculation both in experimental and clinical setting (e.g. intraoperative 
studies assessing hepatic nutritional blood flow during abdominal surgery). Using SDF imaging of the liver in various clinical scenarios could elucidate the regulation and behavior of hepatic microcirculation (e.g. effect of intraoperative fluid therapy, effect of different anesthetics or various types of hepatic and non-hepatic surgical techniques) also with regard to clinical course and patient's outcome. SDF imaging is a reliable powerful noninvasive method with a potential to affect developing new therapeutic strategies that target sinusoidal diameters or FSD based on future experimental and clinical studies assessing physiology and pathophysiology of the liver microcirculation.

\section{Conflict of Interest}

There is no conflict of interest.

\section{Acknowledgements}

Research project MZO 00179906 Arrow International Czech Republic for special custom made SDF probe holder

\section{References}

BAUER M, BAUER I, SONIN NV, KRESGE A, BAVEJA R, YOKOYAMA Y, HARDING D, ZHANG JX, CLEMENS MG: Functional significance of endothelin B receptors in mediating sinusoidal and extrasinusoidal effects of endothelins in the intact rat liver. Hepatology 31: 937-947, 2000.

BOERMA EC, MATHURA KR, VAN DER VOORT PH, SPRONK PE, INCE C: Quantifying bedside-derived imaging of microcirculatory abnormalities in septic patients: a prospective validation study. Crit Care 9: R601-R606, 2005.

ČERNÝ V, TUREK Z, PǍ̌íZKOVÁ R: Orthogonal polarization spectral imaging: a review. Physiol Res 56: 141-147, 2006.

CHUN K, ZHANG J, BIEWER J. Microcirculatory failure determines lethal hepatocyte injury in ischemic/reperfused rat livers. Shock 1: 3-9, 1994.

CLEMENS MG, PAXIAN M, KAMOUN W, ASHBURN J, KRESGE MN, MERKEL S: Regulation of local hepatic oxygen delivery following stress. In: Organ Microcirculation. H ISHII, M SUEMATSU, K TANISHITA, H SUTUKI (eds), Springer-Verlag, Tokyo, 2005, pp 135-144.

DE BACKER D: OPS techniques. Minerva Anesthesiol 69: 388-391, 2003.

DE BACKER D, CRETEUR J, DUBOIS MJ, SAKR Y, VINCENT JL: Microvascular alterations in patients with severe heart failure and cardiogenic shock. Am Heart J 147: 91-99, 2004.

GENZEL-BOROVICZENY O, STROTGEN J, HARRIS AG: Orthogonal polarization spectral imaging (OPS): A novel method to measure the microcirculation in term and preterm infants transcutaneously. Pediatr Res 51: 386-391, 2002.

GRONER W, WINKELMAN JW, HARRIS AG, INCE C, BOUMA GJ, MESSMER K, NADEAU RG: Orthogonal polarization spectral imaging: a new method for study of the microcirculation. Nat Med 5: 1209-1212, 1999.

HARRIS AG, LEIDERER R, PEER F, MESSMER K: Skeletal muscle microvascular and tissue injury after varying durations of ischemia. Am J Physiol 271: H2388-H2398, 1996.

INCE C: Sidestream dark-field (SDF) imaging: an improved technique to observe sublingual microcirculation. Crit Care 8 (Suppl): P72, 2005.

KONDO T, TODOROKI T, HIRANO T, SCHILDBERG FW, MESSMER K. Impact of ischemia-reperfusion injury on dimensional changes of hepatic microvessels. Res Exp Med (Berl) 198: 63-72, 1998.

LANGER S, VON DOBSCHUETZ E, HARRIS AG, KROMBACH F, MESSMER K: Validation of the orthogonal polarization spectral imaging technique on solid organs. In: Orthogonal Polarization Spectral Imaging. K MESSMER (ed), Karger, Basel, 2000, pp 32-46.

LANGER S, HARRIS AG, BIBERTHALER P, VON DOBSCHUETZ E, MESSMER K: Orthogonal polarization spectral imaging as a tool for the assessment of hepatic microcirculation: a validation study. Transplantation 71: 1249-1256, 2001.

LINDERT J, WERNER J, REDLIN M, KUPPE H, HABAZETTL H, PRIES AR: OPS imaging of human circulation: a short technical report. J Vasc Res 39: 368-372, 2002. 
LOVE R, CHOE E, LIPPTON H, FLINT L, STEINBERG S: Positive end-expiratory pressure decreases mesenteric blood flow despite normalization of cardiac output. J Trauma 39: 195-199, 1995.

MARINI JJ, CULVER BN, BUTLER J: Mechanical effect of lung distention with positive pressure on cardiac function. Am Rev Respir Dis 124: 382-386, 1980.

MARZI I, TAKEI I, KNEE J, MENGER MD, GORES GJ, BUHREN V, TRENTZ O, LEMASTERS JJ, THURMAN RG: Assessment of reperfusion injury by intravital fluorescence microscopy following liver transplantation in the rat. Transplant Proc 22: 2004-2005, 1990.

MATHURA KR, INCE C: First clinical use of orthogonal polarization spectral imaging. In: Orthogonal Polarization Spectral Imaging. K MESSMER (ed), Karger, Basel, 2000, pp 94-101.

MATHURA KR, VOLLEBREGT KC, BOER K, DE GRAAFF JC, UBBINK DT, INCE C: Comparison of OPS imaging and conventional capillary microscopy to study the human microcirculation. J Appl Physiol 91: 74-78, 2001.

MENGER MD, MARZI I, MESSMER K. In vivo fluorescence microscopy for quantitative analysis of the hepatic microcirculation in hamsters and rats. Eur Surg Res 23: 158-169, 1991.

PENNINGS FA, INCE C, BOUMA GJ: Continuous real-time visualization of the human cerebral microcirculation during arteriovenous malformation surgery using orthogonal polarization spectral imaging. Neurosurgery 59: 167-171, 2006.

PUHL G, SCHASER KD, PUST D, KOHLER K, VOLLMAR B, MENGER MD, NEUHAUS P, SETTMACHER U: Initial hepatic microcirculation correlates with early graft function in human orthotopic liver transplantation. Liver Transpl 11: 555-563, 2005.

PUHL G, SCHASER KD, VOLLMAR B, MENGER MD, SETTMACHER U: Noninvasive in vivo analysis of the human hepatic microcirculation using orthogonal polarization spectral imaging. Transplantation 75: 756-761, 2003.

SAETZLER RK, JALLO J, LEHR HA, PHILIPS CM, VASTHARE U, ARFORS KE, TUMA RF: Intravital fluorescence microscopy: impact of light-induced phototoxicity on adhesion of fluorescenly labeled leukocytes. J Histochem Cytochem 45: 505-513, 1997.

SAKR Y, DUBOIS MJ, DE BACKER D, CRETEUR J, VINCENT JL: Persistent microvasculatory alterations are associated with organ failure and death in patients with septic shock. Crit Care Med 32: 1825-1831, 2004.

SANER FH, PAVLAKOVIC G, GU Y, PAUL A, RADTKE A, BOCHORN M, FRUHAUF NR, NADALIN S, MALAGO M, BROELSCH CE: Effects of positive end-exspiratory pressure on systemic hemodynamics, with special interest to central venous and common iliac venous pressure in liver transplanted patients. Eur $J$ Anaesth 23: 766-771, 2006.

SCHIESSLER C, SCHAUDIG S, HARRIS AG, CHRIST F: Orthogonal polarization spectral imaging - a new clinical method for monitoring of microcirculation. Anaesthesist 51: 576-579, 2002.

SONIN NV, GARCIA-PAGAN JC, NAKANISHI K: Patterns of vasoregulatory gene expression in the liver response to ischemia/reperfusion and endotoxemia. Shock 11: 175-179, 1999.

TERAJIMA H, KONDO T, ENDERS G, HAMMER C, THIERY J, YAMAMOTO Y, YAMAOKA Y, MESSMER K. Reduction of hepatic microcirculatory failure caused by normothermic ischemia/reperfusion-induced injury by means of heat shock preconditioning. Shock 12: 329-334, 1999.

VOLLMAR B, GLASZ J, LEIDERER R, POST S, MENGER MD. Hepatic microcirculatory perfusion failure is a determinant of liver dysfunction in warm ischemia-reperfusion. Am J Pathol 154: 1421-1431, 1994.

ZHANG JX, PEGOLI W, CLEMENS MG: Endothelin-1 induces direct constriction of hepatic sinusoids. Am J Physiol 266: G624-G632, 1994.

ZHANG JX, BAUER M, CLEMENS MG: Vessel- and target cell-specific actions of endothelin-1 and endothelin-3 in rat liver. Am J Physiol 269: G269-G277, 1995. 